\title{
Mathematical Modeling of the Flight of the Starlings. A Particular Case of an Attractor Repeller Pairs
}

\author{
Araceli Giménez Lorente \\ Higher Education School of Art and Design 1, Plaça de Fadrell, 1, 12002 Castelló de la Plana, Spain
}

Copyright (C) 2019 by authors, all rights reserved. Authors agree that this article remains permanently open access under the terms of the Creative Commons Attribution License 4.0 International License

\begin{abstract}
It presents a mathematical modeling of a biological system, this is a particular case of two fractal sets, an attractor repeller pair, according to the definition given by the Morse-Smalle theory; these are complementary fractal sets, since the complementary of the fractal repeller set is the attractor set, as we can see in the demonstration. The existence of this model in Nature is found in the flight of starlings. This dynamic system works like a beat of a heart, where the movement of the systole is equivalent to the contraction (attractor set) of the flight trajectory of birds and the diastole movement to its expansion (repeller set).The modeling is presented with differential equations written in Matlab code, and several images of the fractal sets associated with the flight pattern of the starlings are generated, so that we can see this model more clearly. We will begin with a definition and its subsequent mathematical demonstration to study this complex system of complementary fractals.
\end{abstract}

Keywords Mathematical Modeling, Biological System, Fractal Sets, Attractor-Repeller Pair, the Flight of Starlings, Matlab Code

\section{Introduction}

The flight of the starlings follows a fractal pattern, the shape is relative to a binary system, in this case an attractor repeller pair. In figure 1 we can a diagram where the blue color represents the attractor and the red color is for the repeller. This diagram represents the phase change of an attractor repeller pair, so we can see the two complementary fractals at the same time.

In this article we will first define the concept attractor repeller pair, with a demonstration we will see that the fractal set called attractor is the complementary set of the repeller, and by means of an algorithm in Matlab code from a differential equations that represent the fractals a mathematical simulation is generated of the flight of the starlings.

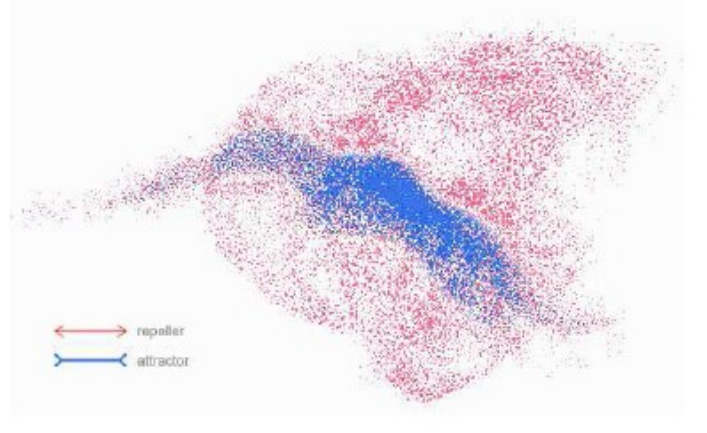

Figure 1. The shape of the flight of the starlings

It will also study the generating points of these pairs of fractals and vortices and their polarity, in relation to the lines of the magnetic field and their mathematical correlation.

This work is part of a great original project, a pioneer in this field.. The system of equations that I have found and its corresponding algorithm written in Matlab code, corresponds to several macroscopic systems that are found in Nature. It is relative to the definition of the Conley theory on invariant sets of attractor repeller pair system such as tornadoes, the flight of starlings, schools of fish, solar storms, geyser system and the beating of a heart.

Once these systems have been studied, the invariants will be observed, which does not change when the system is changed and a theorem will be created. This is the first study in a biological system, which by comparison with an atmospheric phenomenon such as tornadoes gives a lot of relevant information.

\section{Materials and Methods}

The applied scientific method is the observation of the flight pattern of the starlings, its trajectory corresponds to 
chaotic fractals, like the thermodynamics where reality is first observed and then physical laws are written by means of equations, here the applied method is similar, we observe but we check if there is already a mathematical definition of the phenomenon.

In The Morse-Smalle Complex (2005) there is a definition that will be the starting point of this research, binary systems defined as fractal sets attractors-repellers. The important thing of the work is to find them in Nature, and one of these binary systems correspond to the pattern of the flight of the starlings.

First these fractal sets will be defined mathematically, then by means of an algorithm written in Matlab code, mathematical modeling will be carried out, analyzing the images that are generated.

\subsection{Binary system attractor-repeller pair}

The Conley index theory presents a Decompositions of Invariant Sets. A first definition is presented.

Definition 1. Let $\mathrm{S}$ be a compact invariant set. $A \subset S$ is an attractor in $\mathrm{S}$ if there exists a neighborhood $\mathrm{U}$ of $\mathrm{A}$ such that

$$
\omega(U \cap S)=A
$$

The dual repeller of $\mathrm{A}$ in $\mathrm{S}$ is

$$
R:=\{x \in S \mid \omega(x) \cap A=\varnothing\}
$$

The pair $(A, R)$ is called an attractor repeller pair decomposition of S. [1]

\section{Remark: The pair $(\mathrm{A}, \mathrm{R})$}

It is a binary system that is in equilibrium, while the attractor is a convergent set, its complementary the repeller is a divergent set, but together they are a stable system.

We will need other definitions to understand the binary system A-R object of this study.

\section{Definition 2.}

Stable and unstable manifolds. Given a Morse pair (f,g) assume $\mathrm{p}$ is a critical point of $\mathrm{f}$. We define

$$
\begin{aligned}
& W^{-}(p):=\left\{y \in \mathcal{M}: \lim _{t \rightarrow-\infty} \gamma_{y}(t)=p\right\} \\
& W^{+}(p):=\left\{y \in \mathcal{M}: \lim _{t \rightarrow+\infty} \gamma_{y}(t)=p\right\}
\end{aligned}
$$

Where $\gamma_{y}(\cdot)$ denotes the negative gradient flow of $(\mathrm{f}, \mathrm{g})$, and one calls $W^{-}(p)$ the unstable and $W^{+}(p)$ the stable manifold of p.[2]

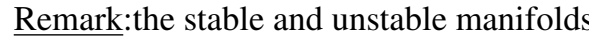
where $W^{-}(p)$ corresponds to the attractor because the function is decreasing and $W^{+}(p)$ is the repeller fractal.

The repeller set assembly corresponds to Spiral Source, as a Type of Critical Point, and the attractor set is a Spiral Sink. [3]

With these bases we will approach mathematically with a demonstration that will imply the complementarity of the complex system A-R, and its importance for this study.

\subsection{A definition with a particular demonstra- tion A-R pair}

The Conley index theory presents this decomposition of Invariant Sets.

Definition 3. Let $\mathrm{S}$ be a compact invariant set. $\mathcal{A} \subset \mathcal{S}$ is an attractor in $\mathrm{S}$ if there exists a neighborhood $\mathrm{U}$ of $\mathrm{A}$ such that

$$
\omega(U \cap S)=A
$$

The dual repeller of $\mathrm{A}$ in $\mathrm{S}$ is

$$
R:=x \in S / \omega(x) \cap A=\varnothing
$$

The pair $(A, R)$

Let $(A, R)$ pair descomposition of $S$

$$
\Rightarrow S=A \cup R \cup C(R, A)
$$

where $C(R, A):=x \in S / \omega(x) \subset A, \alpha(x) \subset R$

$$
\begin{gathered}
\exists V: S \rightarrow[0,1] / \\
1 . R=V^{-1}(1) \\
2 . A=V^{-1}(1)
\end{gathered}
$$

3. if $x \in C(A, R) \wedge t>0 \rightarrow v(x)=V(Q(t, x))$

$\varphi_{\lambda}: R \times R^{n} \rightarrow R^{n}, \lambda \in[-1,1]$

$N \subset R^{n}$ compact set

$$
S_{\lambda}=\operatorname{Inv}\left(N, \varphi_{\lambda}\right) S_{\lambda_{0}}, S_{\lambda_{1}} \Rightarrow \varphi_{\lambda}, \lambda \in\left[\lambda_{0}, \lambda_{1}\right] \subset[-1,1]
$$

\section{Demo.}

$$
R:=x \in S / w(x) \cap A=\varnothing
$$

Then $A:=x \in S / \alpha(x) \cap A \neq \varnothing ? \Rightarrow R^{C}=A$ ? 


$$
\begin{aligned}
& \Rightarrow R^{C}=x \in S / \neg \omega(x) \cap A=\varnothing \\
& \stackrel{\omega(x) \subset A \wedge A=\omega(U \cap S)}{\longrightarrow} R^{C}=x \in S / \alpha(x) \cap A \neq \varnothing \\
& \underset{C(R, A):=x \in S / \omega(x) \subset A, \alpha(x) \subset R}{\stackrel{S=A \cup R \cup C(R, A)}{\underset{ }{C}}} R^{C}=x \in S / \alpha(x) \underbrace{\omega(U \cap S)}_{A} \neq \\
& \rightsquigarrow A \Rightarrow R^{C}=A \square
\end{aligned}
$$

\section{Results}

The initial model of the repeller fractal is based on plant growth, in particular is the function that describes how to grow a leaf of a deciduous tree. In polar coordinates we have the following function.

$$
f_{1}=\theta \cdot \sin (\theta)
$$

It is a finite 'fractal object', because it has been associated with a natural phenomenon which is born and dies like all living beings. With the origin and its derivative function we will build the two fractals, the attractor-repeller pair. [5]

Once we have the equations and the algorithm corresponding to the two complementary fractals, we pass to the writing in Matlab code of said algorithm corresponding to the recursive pattern of the flight of the starlings.

\subsection{Math modeling. A particular case in a biological sys- tem}

This is a mathematical modeling in Matlab code.

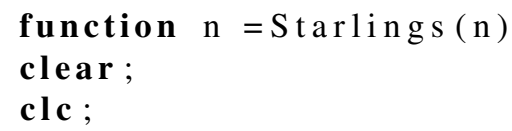

theta $=0: 0.01: 20 * \mathbf{p i} ;$

delta $=-20 * \mathbf{p i}: 0.01: 0$;

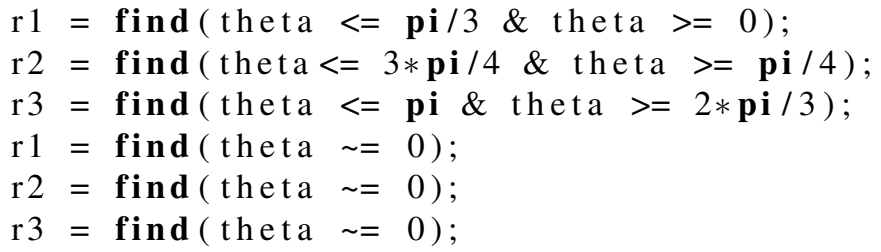

$\%$ Attractor

$\mathrm{t}(\mathrm{r} 1)=-\operatorname{delta}(\mathrm{r} 1) . * \sin (\operatorname{delta}(\mathrm{r} 1))$;

$\mathrm{v}(\mathrm{r} 2)=\cos (\operatorname{delta}(\mathrm{r} 2))$;

$\mathrm{w}(\mathrm{r} 3)=2 * \mathbf{p i} *(\mathrm{t}(\mathrm{r} 1) . * \mathrm{v}(\mathrm{r} 2))+(\operatorname{delta}(\mathrm{r} 3)) ;$

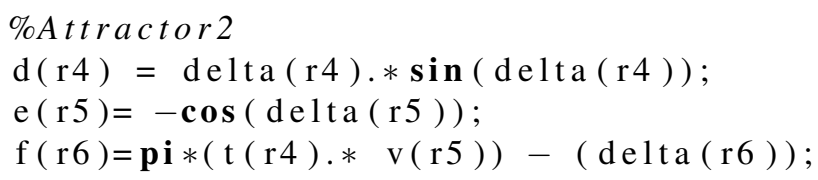

hold on

grid on

$\operatorname{plot} 3\left(\mathrm{x}, \mathrm{y}, \mathrm{z},{ }^{\prime} \mathrm{r}\right.$ ')

view (3)

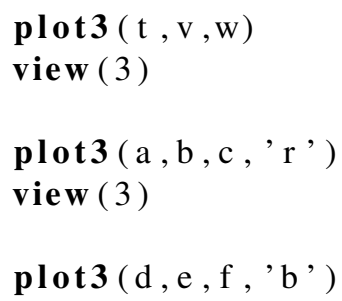


end

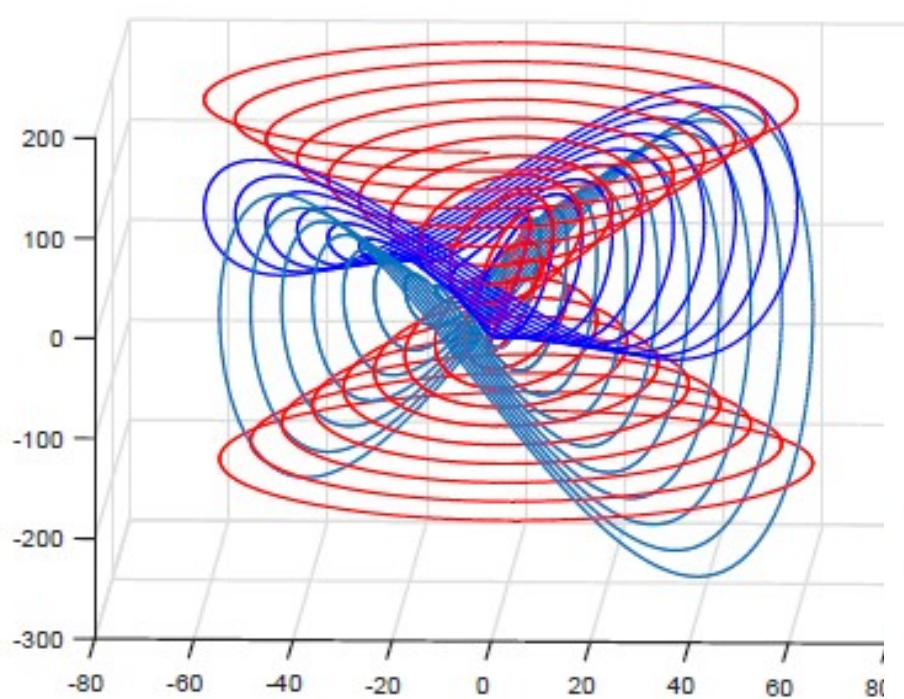

Figure 2. Math modeling in Matlab code

Where the red color represents the repeller and the blue colors are relative to the strange attractors.

We have the binary attractor-repeller system. In the modeling appear two systems, are two attractors and two repellers.

\subsection{Study of the vortices generated in the binary system}

This mathematical study can be completed with the relationship between the behavior of the magnetic field, and the generation points (analogous to the magnetic poles) of the binary system A-R.

Figures 7 and 8 visually explain this relationship of polarity and the points of generation of the fractals studied here, what are the vortices that generate our binary system A-R.

Although the vortex concept is a model related to fluid mechanics, how this research is strictly mathematical, a correlation with the magnetic field lines has been sought.

A symmetric solutions is in relation to symmetric configurations than correspond to solutions where either $x_{1}=-x_{2}$ or $r_{12}=2$. Both cases are simple enough to analyze using Gröbner bases.

Case 1: $x_{1}=-x_{2}[6]$

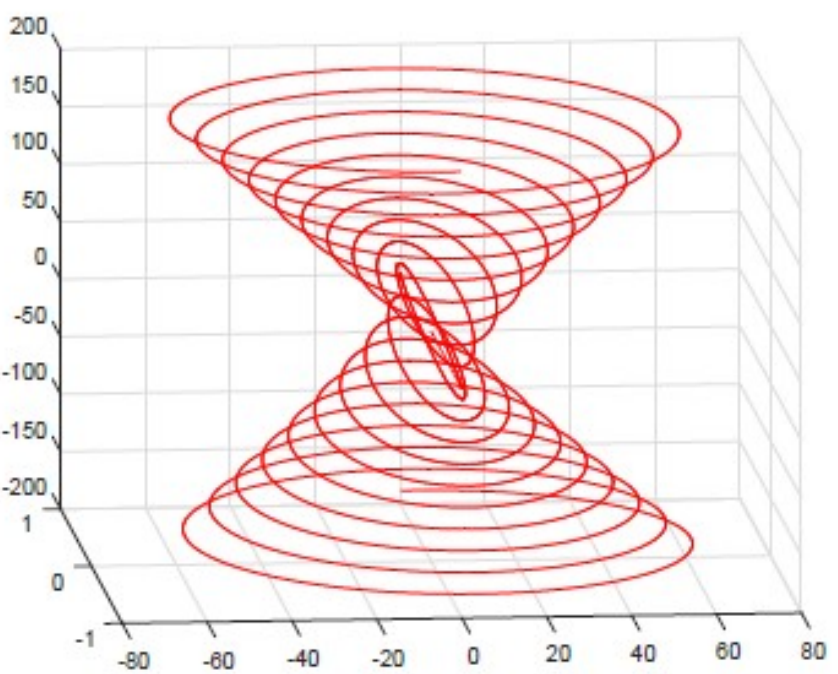

Figure 3. System with two repellers

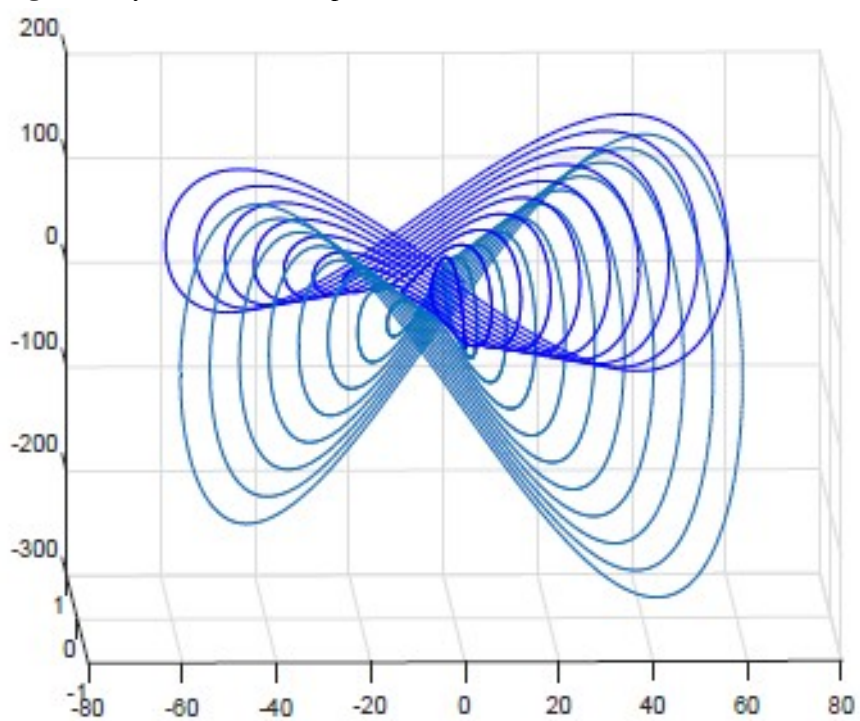

Figure 4. System wiht two attractors 


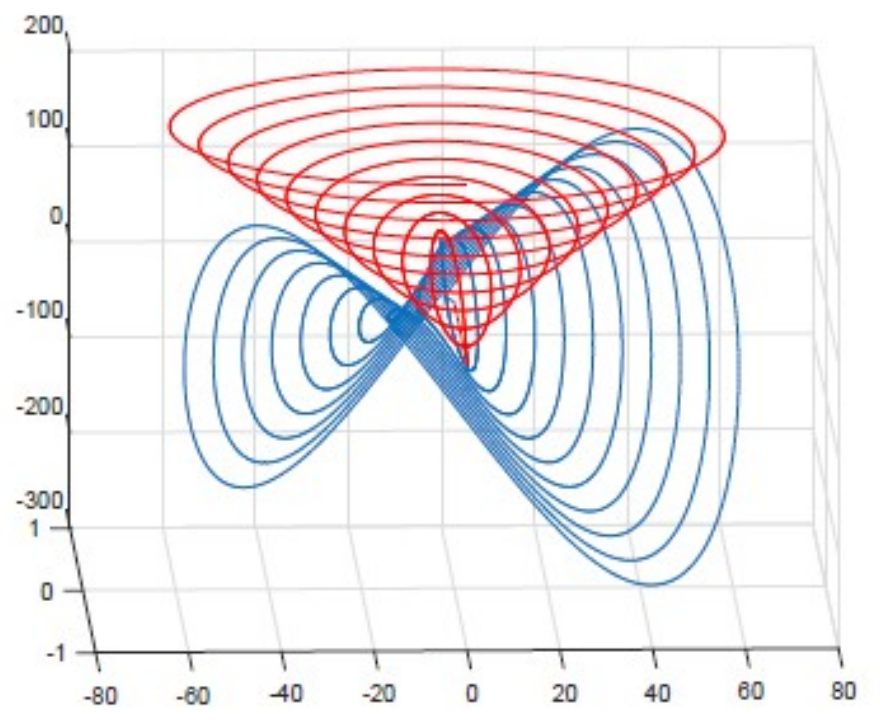

Figure 5. System with a attractorrepeller pair

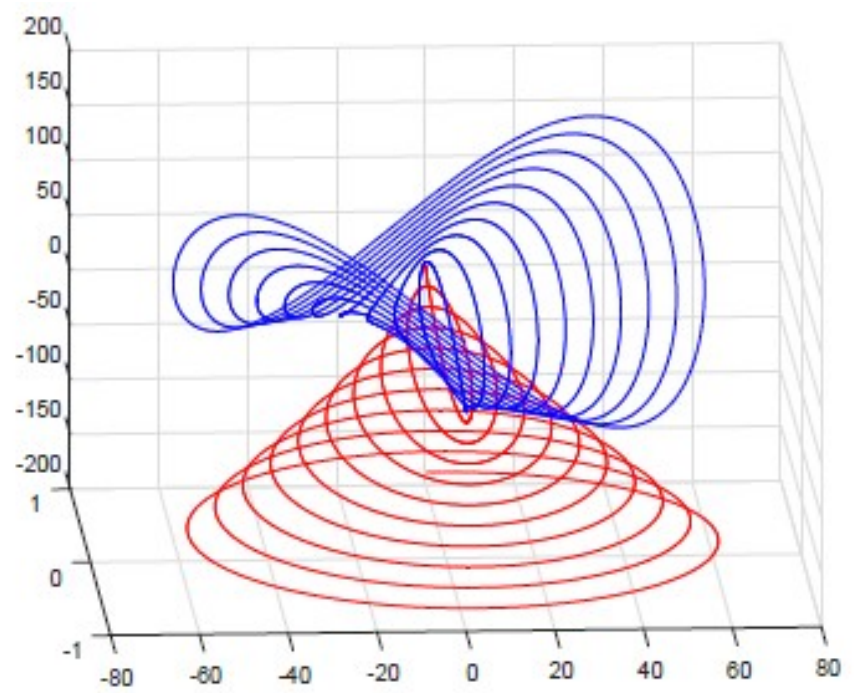

Figure 6. System with a attractorrepeller pair (symmetry)
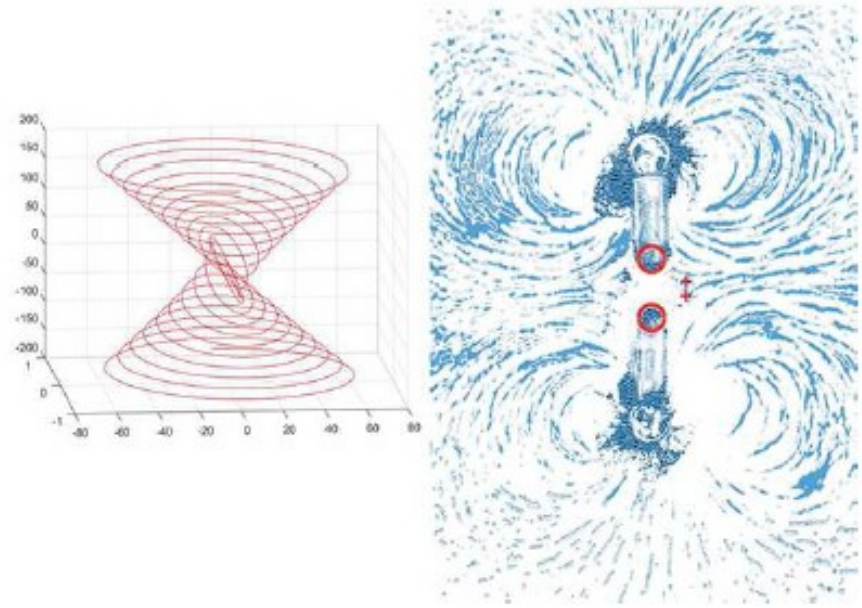

Figure 7. Double polarity in two repellers, comparative with magnetism
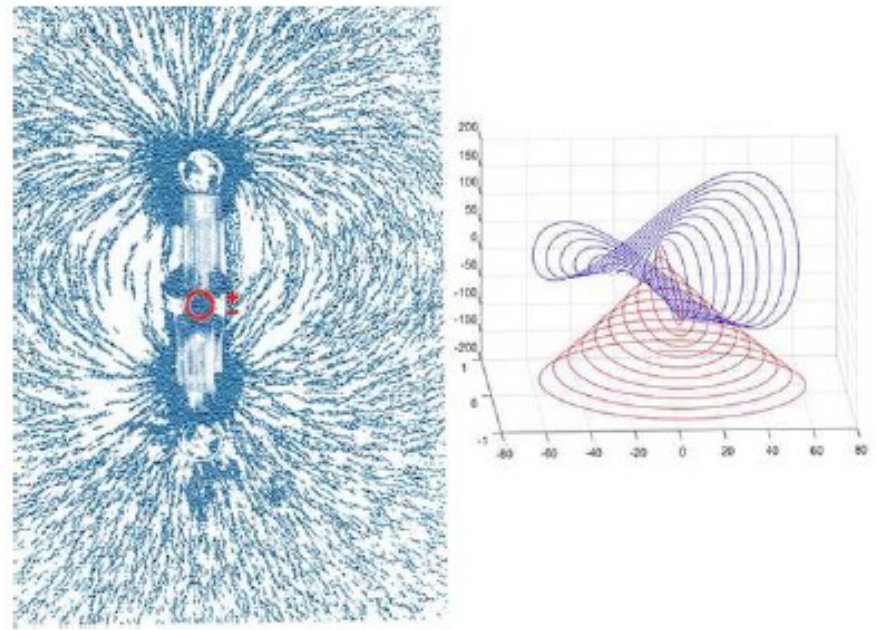

Figure 8. Double polarity in attractor repellers pair, comparative with magnetism 
When extrapolated this case that the center of vorticity $\mathrm{c}$ is at the origin, In a real case that is represented in figure 8 . In this case, the two fractal are different sign, the attractor is negative and the repeller is positive in relation to polarity; iff $c \in[-1,1[\mathrm{c}$ is the center of the vortex and in this case there is only one vortex (figure 8).

In the second case, we have the same polarity, it is the case of two attractors -- or two repellers ++ , there are two collinear vortex (figure 7 .

There is a complex case we have the 2 - vortex pair systems theorem concerning the problem of the four vortices.

Theorem: 4-vortex 1. When $m \in(-1,0)$, the signs of the vorticities in a collinear relative equilibrium must be arranged as +--+ symmetric case only, or as +-+- or -+-+ when asymmetric solutions exist. Therefore, when $m<0$, it is not possible to have a collinear solutions where both pairs of vortices with the same strength are adjacent to each other. [6]

These will be the cases where the generating points of the vortices of our A-R even fractals are linked in several fractals, being the collinear or adjacent vortices, symmetrical or asymmetric. But this analysis is so complex that we will leave it for another mathematical study. Simply the existence of this possibility is exposed

\section{Discussion}

The dynamics of fluids have not been taken into account for this study, since it is an exclusively mathematical work, based on algebraic topology and fractal geometry.

As the fractal models usually only involve attractors, and the concept of repeller is used, we wanted to give a demonstration not only of its existence, but also that a repeller set is the inverse of an attractor set, being faithful to the definition of the Conley index theory.

In spite of this, it has been extended to the study of vortices in correlation with magnetic fields, since they are similar in terms of structure and polarity relations; they also have an implicit correlation because the flight of the starlings responds to geomagnetic navigation.

It is the first time that this phenomenon is studied in a biological system, such as the flight of starlings, it could also be extrapolated to the movement of the fish bank, among other similar systems.

To do this study besides being based on the direct observation of the flight of these birds, I have seen videos that are referenced [6],[7], and has begun by making a diagram that represents the phase of change of the binary attractor-repeller system (figure 1).
The phase change shown in figure 1 is the best visual evidence of the binary attractor repeller system. To perform the modeling it has been necessary to extract the differential equations of both systems to then perform an algorithm in Matlab code that is the final result, mathematical modeling in Figure 2.

The methodology used can be extrapolated to other systems such as solar storms, the geyser-boiler system or even the beating of a heart.

\section{Conclusions}

Benoit Mandelbrot discovered fractal patterns in nature. It is time to go a step further, finding a binary system of complementary fractals that can be extrapolated to the chaotic flight of starlings. In this article we want to explain the logical process by which we arrive at the conclusion that the flight of the starlings correspond to the definition of attractor repeller pairs. This chaotic system appears in equilibrium, since they are complementary and non-contradictory fractal sets.The algorithm in Matlab code generates a modeling that is necessary to understand the system of differential equations that allude to the attractor repeller pair.

This is just a mathematical study, fluid dynamics do not intervene in modeling, so this modeling can extrapolated to other particular cases, to other biological systems, such as the movement of fish schools. That the fluid where the fractal trajectory of the carbon unit develops, or be alive, whether air or water does not interfere in the final form, since we are talking about algebraic topology, not classical geometry, the shape can be change but the essence remains.

\section{Acknowledgements}

I thank Ph.Doctor Francisca Mascaró for believing in my potential as a mathematician and as a mentor to support me, thank Ph. Doctor Gustau Camps i Valls because I introduced in Matlab programming that allowed me to do my math modeling, and would also like to thank my friend José Luis Rodríguez Blancas, who has always believed in me and has supported me as a mathematician.

\section{REFERENCES}

[1] Konstantin Mischaikow.Marian Mrozek. Conley Index Theory. Preprint. p.7. 1999.

[2] Florian Schätz. The Morse-Smalle Complex. Magister der Naturwissenschaften an der Universität Wien, Preprint. 
p.16.Wien, Österreich. 2005.

[3] Ryan, Shawn D.. 9.1 The Phase Plane: Linear Systems. Lecture Notes for Math 251: ODE and PDE. Lecture 27. p. 7. Spring 2012.

[4] Konstantin Mischaikow.The Conley Index Theory:A Brief Introduction. Banach Center Publication, Volume 47 Institute of Mathematics Polish Academy of Sciences WARSZAWA. Georgia Institute of Technology Atlanta, Georgia. U.S.A. 30332, p.12. U.S.A. 1999.

[5] Araceli Giménez Lorente. Simulation of a Tornadoes as a Particular Case of an Attractor-repeller Pairs. Universal Journal of Physics and Application 9(6) : 251 - 257, 2015 http://www.hrpub.org.
[6] 1 Hampton, Marshal;Roberts, Gareth E; Santopetre, Manuele. Relative Equilibria in the Four-Vortex Problems with Two Pairs of Equal Vortices. ArXiv:1208.4204v1 [math.CA]. p. 19, 23. 21 Aug2012.

[7] National Geographic. Flight of the Starlings: Watch This Eerie but Beautiful Phenomenon I Short Film Showcase, 15 nov. 2016. Online Available: https://www.youtube.com/watch? $v=V 4 f \_1 \_r 80 R Y$.

[8] John Downer Productions. Ultimate murmuration-Peregrine hunts starlings in RomeEarthflight. January 2012. Online Available: https://www.youtube.com/watch? $v=V-m C u F Y f J d I$. 